Honam Mathematical J. 34 (2012), No. 1, pp. 093-101

http://dx.doi.org/10.5831/HMJ.2012.34.1.93

\title{
THE NUMBER OF POINTS ON ELLIPTIC CURVES $E_{A}^{0}: y^{2}=x^{3}+A x$ OVER $\mathbb{F}_{p}$ MOD 24
}

\author{
Hwasin Park, Soonho You, Daeyeoul Kim and Minhee Kim
}

\begin{abstract}
Let $E_{A}^{B}$ denote the elliptic curve $E_{A}^{B}: y^{2}=x^{3}+A x+B$. In this paper, we calculate the number of points on elliptic curves $E_{A}^{0}: y^{2}=x^{3}+A x$ over $\mathbb{F}_{p} \bmod 24$. For example, if $p \equiv 1(\bmod 24)$ is a prime, $3 t^{2} \equiv 1(\bmod p)$ and $A(-1+2 t)$ is a quartic residue modulo $p$, then the number of points in $E_{A}^{0}: y^{2}=x^{3}+A x$ is congruent to 0 modulo 24 .
\end{abstract}

\section{Introduction}

Let $p>3$ be a prime, and let $\mathbb{F}_{p}$ be the finite field of $p$ elements. From now on we let $E_{A}^{B}$ denote the elliptic curve $y^{2}=x^{3}+A x+B$ over $\mathbb{F}_{p}$ where $A, B \in \mathbb{F}_{p}$. The set of points $(x, y) \in \mathbb{F}_{p} \times \mathbb{F}_{p}$ together with a point $O$ at infinity is called the set of points of $E_{A}^{B}$ in $\mathbb{F}_{p}$ and is denoted by $E_{A}^{B}\left(\mathbb{F}_{p}\right)$. And let $\# E_{A}^{B}\left(\mathbb{F}_{p}\right)$ be the cardinality of the set $E_{A}^{B}\left(\mathbb{F}_{p}\right)$. For a more detailed information about elliptic curves in general, see [Si]. It has been always interesting to look for the number of points over a given field $\mathbb{F}_{p}$. In $[\mathrm{S}]$, three algorithms to find the number of points on an elliptic curve over a finite field are given. Also in [DISC], [DSC], [ISDC2] the number of rational points on Frey elliptic curves $E: y^{2}=x^{3}-n^{2} x$ and $E: y^{2}=x^{3}+a^{3}$ are found.

In 2003, H. Park, D. Kim and H. Lee calculated the number of points on elliptic curves $E_{A}^{0}: y^{2}=x^{3}+A x$ over $\mathbb{F}_{p} \bmod 8$ ([PKL], [ISDBC]). The purpose of this paper is to give a straightforward calculation of the number mod 24 of points on elliptic curves over a finite field.

In this article, we derive a type of generalization of their results ([PKL], [ISDBC]) by means of elliptic curves mod 24.

Received December 29, 2011. Accepted February 7, 2012. 2000 Mathematics Subject Classification. 11A15, 11G07.

Key words and phrases. elliptic curves. 


\section{The number of points on elliptic curves $E_{A}^{0}$ : $y^{2}=x^{3}+A x$ over $\mathbb{F}_{p} \bmod 24$}

Let $p$ be a prime, and let $t$ be an element of $\mathbb{F}_{p}^{*}=\mathbb{F}_{p}-\{0\}$ such that $3 t^{2} \equiv 1(\bmod p)$. Then such an element $t$ exists when $p \equiv 1,11$ $(\bmod 12)$. Now, for simplicity we set

$q_{4}:$ quartic residue in $\mathbb{F}_{p}$,

$q_{2}$ : quadratic residue but quartic non-residue in $\mathbb{F}_{p}$,

$q_{1}$ : quadratic non-residue in $\mathbb{F}_{p}$ and

$\left(\frac{a}{p}\right)_{3}=1$ if $x^{3} \equiv a(\bmod p)$ is solvable.

Theorem 2.1. 1. If $p \equiv 1,11(\bmod 12)$ is a prime and $3 t^{2} \equiv 1$ $(\bmod p)$, then we get the following table.

\begin{tabular}{|c|c|c|c|c|}
\hline$p$ & $A$ & $-1 \pm 2 t$ & $A(-1 \pm 2 t)$ & $\# E_{A}^{0}\left(\mathbb{F}_{p}\right)$ \\
\hline \multirow{5}{*}{$1(\bmod 24)$} & $q_{4}$ & $q_{4}$ & $q_{4}$ & $0(\bmod 24)$ \\
\cline { 2 - 5 } & $q_{4}$ & $q_{2}$ & $q_{2}$ & 8 or $16(\bmod 24)$ \\
\cline { 2 - 5 } & $q_{4}$ & $q_{1}$ & $q_{1}$ & 8 or $16(\bmod 24)$ \\
\cline { 2 - 5 } & $q_{2}$ & $q_{2}$ & $q_{4}$ & $12(\bmod 24)$ \\
\cline { 2 - 5 } & $q_{2}$ & $q_{4}$ & $q_{2}$ & 4 or $20(\bmod 24)$ \\
\cline { 2 - 5 } & $q_{2}$ & $q_{1}$ & $q_{1}$ & 4 or $20(\bmod 24)$ \\
\cline { 2 - 5 } & $q_{1}$ & $q_{1}$ & $q_{4}$ & $18(\bmod 24)$ \\
\cline { 2 - 5 } & $q_{1}$ & $q_{1}$ & $q_{2}$ & 2 or $10(\bmod 24)$ \\
\cline { 2 - 5 } & $q_{1}$ & $q_{4}$ & $q_{1}$ & 2 or $10(\bmod 24)$ \\
\cline { 2 - 5 } & $q_{1}$ & $q_{2}$ & $q_{1}$ & 2 or $10(\bmod 24)$ \\
\hline $11(\bmod 24)$ & & & $a l l$ & $12(\bmod 24)$ \\
\hline \multirow{5}{*}{$(\bmod 24)$} & $q_{4}$ & $q_{4}$ & $q_{4}$ & $12(\bmod 24)$ \\
\cline { 2 - 5 } & $q_{4}$ & $q_{2}$ & $q_{2}$ & 4 or $20(\bmod 24)$ \\
\cline { 2 - 5 } & $q_{4}$ & $q_{1}$ & $q_{1}$ & 4 or $20(\bmod 24)$ \\
\cline { 2 - 5 } & $q_{2}$ & $q_{2}$ & $q_{4}$ & $0(\bmod 24)$ \\
\cline { 2 - 5 } & $q_{2}$ & $q_{1}$ & $q_{2}$ & 8 or $16(\bmod 24)$ \\
\cline { 2 - 5 } & $q_{1}$ & $q_{1}$ & $q_{1}$ & 8 or $16(\bmod 24)$ \\
\cline { 2 - 5 } & $q_{1}$ & $q_{1}$ & $q_{2}$ & 2 or $10(\bmod 24)$ \\
\cline { 2 - 5 } & $q_{1}$ & $q_{4}$ & $q_{1}$ & 2 or $10(\bmod 24)$ \\
\cline { 2 - 5 } & $q_{1}$ & $q_{2}$ & $q_{1}$ & 2 or $10(\bmod 24)$ \\
\hline $23(\bmod 24)$ & & & all & $0(\bmod 24)$ \\
\hline
\end{tabular}

2. If $p \equiv 5,7(\bmod 12)$ is a prime, then we get the following table. 


\begin{tabular}{|c|c|c|}
\hline$p$ & $A$ & $\# E_{A}^{0}\left(\mathbb{F}_{p}\right)$ \\
\hline \multirow{3}{*}{$5(\bmod 24)$} & $q_{4}$ & 4 or $20(\bmod 24)$ \\
\cline { 2 - 3 } & $q_{2}$ & 8 or $16(\bmod 24)$ \\
\cline { 2 - 3 } & $q_{1}$ & 2 or $10(\bmod 24)$ \\
\hline $7(\bmod 24)$ & all & $8(\bmod 24)$ \\
\hline \multirow{3}{*}{$17(\bmod 24)$} & $q_{4}$ & 8 or $16(\bmod 24)$ \\
\cline { 2 - 3 } & $q_{2}$ & 4 or $20(\bmod 24)$ \\
\cline { 2 - 3 } & $q_{1}$ & 2 or $10(\bmod 24)$ \\
\hline $19(\bmod 24)$ & all & $20(\bmod 24)$ \\
\hline
\end{tabular}

To prove this theorem, we need the following propositions and lemmas.

Proposition $2.2([\mathrm{~K}]$ p.145, [Si] p.323).

1. Let $p \neq 2,3$. Then $E_{A}^{B}$ is supersingular if and only if $\# E_{A}^{B}=p+1$.

2. If $p \equiv 3(\bmod 4)$ is a prime and $E_{A}^{0}: y^{2}=x^{3}+A x$ is an elliptic curve over $\mathbb{F}_{p}$, then $\# E_{A}^{0}=p+1$.

3. If $p \equiv 2(\bmod 3)$ is a prime and $E_{0}^{B}: y^{2}=x^{3}+B$ is an elliptic curve over $\mathbb{F}_{p}$, then $\# E_{0}^{B}=p+1$.

By Proposition $2.2(2)$, if $p \equiv 7,11(\bmod 12)$ is a prime and $E_{A}^{0}$ : $y^{2}=x^{3}+A x$ is an elliptic curve over $\mathbb{F}_{p}$ then $\# E_{A}^{0}=p+1$. So, we consider the elliptic curve $E_{A}^{0}: y^{2}=x^{3}+A x$ when $p \equiv 1,5(\bmod 12)$.

Proposition $2.3([\mathrm{KKP}])$. Let $E_{A}^{B}: y^{2}=x^{3}+A x+B$ be an elliptic curve over $\mathbb{F}_{p}$ and $P=(x, y)$ be a point in $E_{A}^{B}\left(\mathbb{F}_{p}\right)$ which is not a point at infinity, where $E_{A}^{B}\left(\mathbb{F}_{p}\right)$ is the group of points on $E$. Then the followings are equivalent

1. $P=(x, y)$ is a point of order 3 in $E_{A}^{B}\left(\mathbb{F}_{p}\right)$.

2. $3 x^{4}+6 A x^{2}+12 B x-A^{2}$ is congruent to 0 to modulo $p$.

We denoted by $N_{p}(f(x))$ the number of solutions of the congruence equation $f(x) \equiv 0(\bmod p)$. Let $(\because)$ be the Legendre symbol and let $D=a_{1}^{2} a_{2}^{2}-4 a_{2}^{3}-4 a_{1}^{3} a_{3}-27 a_{3}^{2}+18 a_{1} a_{2} a_{3}$ be the discriminant of the cubic polynomial $x^{3}+a_{1} x^{2}+a_{2} x+a_{3}$.

Lemma 2.4. If $p>3$ is a prime, $a_{1}, a_{2}, a_{3} \in \mathbb{Z}$ and $p \nmid D$, then

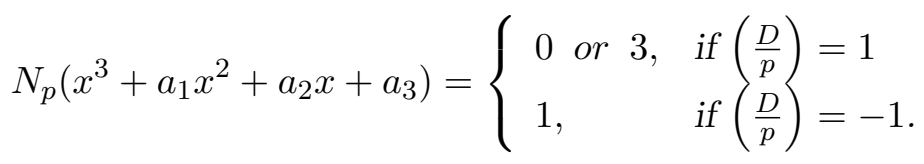


Proof. See Cohen [C, pp.198-199], Dickson [D] or Stickelberger [St].

Proposition 2.5 ([IR]). Let $p$ and $q$ be odd primes. Then the followings are satisfied.

1. If $p \equiv 1(\bmod 4)$, then $\left(\frac{-1}{p}\right)=1$.

2. If $p \equiv \pm 1(\bmod 12)$, then $\left(\frac{3}{p}\right)=1$.

Proposition 2.6 ([ISDBC], [PKL]). If $p$ is a prime, then when $p \equiv 1(\bmod 8)$,

$\# E_{A}^{0}= \begin{cases}0(\bmod 8) & \text { if } A \text { is a quartic residue in } \mathbb{F}_{p} \\ 4(\bmod 8) & \text { if } A \text { is quadratic residue but quartic non-residue in } \mathbb{F}_{p} \\ 2(\bmod 8) & \text { if } A \text { is quadratic non-residue in } \mathbb{F}_{p}\end{cases}$

and when $p \equiv 5(\bmod 8)$,

$\# E_{A}^{0}= \begin{cases}4(\bmod 8) & \text { if } A \text { is a quartic residue in } \mathbb{F}_{p} \\ 0(\bmod 8) & \text { if } A \text { is quadratic residue but quartic non-residue in } \mathbb{F}_{p} \\ 2(\bmod 8) & \text { if } A \text { is quadratic non-residue in } \mathbb{F}_{p} .\end{cases}$

Example 2.7. Let $p=5$. Then we get the following.

\begin{tabular}{|c|c|c|}
\hline$A$ & $q_{i}$ & $\# E_{A}^{0}\left(\mathbb{F}_{5}\right)$ \\
\hline 1 & $q_{4}$ & 4 \\
\hline 2 & $q_{1}$ & 2 \\
\hline 3 & $q_{1}$ & 10 \\
\hline 4 & $q_{2}$ & 8 \\
\hline
\end{tabular}

Now, we will give the results concerning $\# E_{A}^{0}$ over $\mathbb{F}_{p}$ modulo 3 .

Lemma 2.8. Let $E_{A}^{0}: y^{2}=x^{3}+A x$ be an elliptic curve over $\mathbb{F}_{p}$. If $\left(\frac{3}{p}\right)=-1$, then $\# E_{A}^{0} \not \equiv 0(\bmod 3)$.

Proof. Assume that $\# E_{A}^{0} \equiv 0(\bmod 3)$. Then there exists a point $P=(x, y) \in E_{A}^{0}\left(\mathbb{F}_{p}\right)$ satisfying $3 P=O$. By Proposition 2.3 , we deduce that $f(x)=3 x^{4}+6 A x^{2}-A^{2} \equiv 0(\bmod p)$, and hence $3\left(x^{2}+A\right)^{2} \equiv 4 A^{2}$ $(\bmod p)$. We easily check that $\left(\frac{\left(x^{2}+A\right)^{2}}{p}\right)=\left(\frac{4}{p}\right)=\left(\frac{A^{2}}{p}\right)=1$, and $\left(\frac{3}{p}\right)=-1$ by assumption. It is a contradiction. So, $\# E_{A}^{0} \not \equiv 0(\bmod 3)$.

Corollary 2.9. Let $E_{A}^{0}: y^{2}=x^{3}+A x$ be an elliptic curve over $\mathbb{F}_{p}$. If $p \equiv 5,7(\bmod 12)$ are primes, then $\# E_{A}^{0} \not \equiv 0(\bmod 3)$. 
Proof. Since $\left(\frac{3}{5}\right)=\left(\frac{3}{7}\right)=-1$, we derive that $\# E_{A}^{0} \not \equiv 0(\bmod 3)$, by Lemma 2.8.

Example 2.10. Let $p=7$.

If $A=1$, then $\# E_{1}^{0}: y^{2}=x^{3}+x=16 \not \equiv 0(\bmod 3)$.

If $A=2$, then $\# E_{2}^{0}: y^{2}=x^{3}+2 x=20 \not \equiv 0(\bmod 3)$.

If $p \equiv 5,7(\bmod 12)$, then $\left(\frac{3}{p}\right)=-1$ and $3 t^{2} \not \equiv 1(\bmod p)$. Now, we will consider the cases when $p \equiv 1,11(\bmod 12)$.

Lemma 2.11. Let $3 t^{2} \equiv 1(\bmod p)$ with $t \in \mathbb{F}_{p}$. Then

$$
\left(\frac{-1+2 t}{p}\right)=\left\{\begin{array}{lll}
\left(\frac{-1-2 t}{p}\right) & \text { if } p \equiv 1 \quad(\bmod 12) \\
-\left(\frac{-1-2 t}{p}\right) & \text { if } p \equiv 11 \quad(\bmod 12) .
\end{array}\right.
$$

Proof. Since $\left(\frac{-1+2 t}{p}\right)\left(\frac{-1-2 t}{p}\right)=\left(\frac{1-4 t^{2}}{p}\right)=\left(\frac{1-3 t^{2}-t^{2}}{p}\right)=\left(\frac{-t^{2}}{p}\right)=$ $\left(\frac{-1}{p}\right)\left(\frac{t^{2}}{p}\right)=\left(\frac{-1}{p}\right)$, we get the result by Proposition $2.5(1)$.

Lemma 2.12. Let $p \equiv 1(\bmod 12)$ be a prime and let $3 t^{2} \equiv 1$ $(\bmod p)$ with $t \in \mathbb{F}_{p}^{*}$. Then $\# E_{A}^{0}: y^{2}=x^{3}+A x \equiv 0(\bmod 3)$ if and only if $-A \pm 2 t A$ are quartic residues in $\mathbb{F}_{p}$.

Proof. Assume that $-A \pm 2 t A$ are quartic residues in $\mathbb{F}_{p}$.

Let $x^{2} \equiv-A \pm 2 t A(\bmod p)$, where $x$ is a quadratic residue in $\mathbb{F}_{p}$. Also, we have $x^{2}+A \equiv \pm 2 t A(\bmod p)$ and $\left(x^{2}+A\right)^{2} \equiv t^{2}(2 A)^{2}(\bmod p)$. It follows from $3 t^{2} \equiv 1(\bmod p)$ and $3\left(x^{2}+A\right)^{2} \equiv 4 A^{2}(\bmod p)$ that

$$
3 x^{4}+6 A x^{2}-A^{2} \equiv 0 \quad(\bmod p) .
$$

Here, we put $f(x)=3 x^{4}+6 A x^{2}-A^{2}$. Since $3 t^{2} \equiv 1(\bmod p)$, $\left(\frac{2 t}{p}\right)\left(\frac{2 t-1}{p}\right)=\left(\frac{4 t^{2}-2 t}{p}\right)=\left(\frac{t^{2}-2 t+3 t^{2}}{p}\right)=\left(\frac{t^{2}-2 t+1}{p}\right)=\left(\frac{(t-1)^{2}}{p}\right)=1$. That is, $\left(\frac{2 t}{p}\right)=\left(\frac{2 t-1}{p}\right)$. Similarly, $\left(\frac{-2 t}{p}\right)=\left(\frac{-2 t-1}{p}\right)$. Since $x^{2} \equiv$ $-A \pm 2 t A(\bmod p),\left(\frac{-1 \pm 2 t}{p}\right)=\left(\frac{A}{p}\right)$. By Lemma 2.11 we know that $\left(\frac{-1+2 t}{p}\right)=\left(\frac{-2 t-1}{p}\right)=\left(\frac{2 t}{p}\right)=\left(\frac{-2 t}{p}\right)=\left(\frac{A}{p}\right)$. So we deduce that

$$
\left(\frac{ \pm 2 t A}{p}\right)=1 \text {. }
$$


Therefore, there exists a point $P(x, y)$ in $E_{A}^{0}: y^{2}=x^{3}+A x=x\left(x^{2}+\right.$ $A)=x( \pm 2 t A)$ such that $f(x) \equiv 0(\bmod p)$, because $x$ and $\pm 2 t A$ are quadratic residues in $\mathbb{F}_{p}$. That is, $\# E_{A}^{0}: y^{2}=x^{3}+A x \equiv 0(\bmod 3)$ by Proposition 2.3.

Conversely, we assume that $\# E_{A}^{0}: y^{2}=x^{3}+A x \equiv 0(\bmod 3)$. We first consider the case where $-A \pm 2 t A$ are quartic non-residues in $\mathbb{F}_{p}$. Since $\# E_{A}^{0}: y^{2}=x^{3}+A x \equiv 0(\bmod 3), 3 x^{4}+6 A x^{2}-A^{2} \equiv 0(\bmod p)$, by Proposition 2.3. Then, $x^{2} \equiv-A \pm 2 t A(\bmod p)$ by $(2.0 .1)$.

Now, if $-A \pm 2 t A$ are quadratic non-residues in $\mathbb{F}_{p}$, then there does not exist a point $P(x, y)$ of $E_{A}^{0}: y^{2}=x^{3}+A x$ such that $f(x) \equiv 0(\bmod p)$. Finally, if $-A \pm 2 t A$ are quadratic residues but quartic non-residues in $\mathbb{F}_{p}$, then $x$ is a quadratic non-residue. And since $\pm 2 t A$ are quadratic residues in $\mathbb{F}_{p}$ by $(2.0 .2), x( \pm 2 t A)$ are quadratic non-residues in $\mathbb{F}_{p}$. Consequently, there does not exist a point $P(x, y)$ of $E_{A}^{0}: y^{2}=x^{3}+A x$ such that $f(x) \equiv 0(\bmod p)$. Therefore, if $-A \pm 2 t A$ are quartic nonresidues in $\mathbb{F}_{p}$, then $\# E_{A}^{0}: y^{2}=x^{3}+A x \not \equiv 0(\bmod 3)$.

Example 2.13. Let $p=13$. Then roots of $3 t^{2} \equiv 1(\bmod 13)$ are $t= \pm 3$ and $-1 \pm 2 t=5,6$. Then we get the following table.

\begin{tabular}{|c|c|c|}
\hline$A$ & $-A \pm 2 t A$ & $\# E_{A}^{0}\left(\mathbb{F}_{13}\right)$ \\
\hline $7,8,11$ & $q_{4}$ & 18 \\
\hline $2,5,6$ & $q_{2}$ & 10 \\
\hline $1,3,9$ & $q_{1}$ & 20 \\
\hline $4,10,12$ & $q_{1}$ & 8 \\
\hline
\end{tabular}

And let $p=37$. Then roots of $3 t^{2} \equiv 1(\bmod 37)$ are $t= \pm 5$ and $-1 \pm 2 t=9,26$. Then we get the following table.

\begin{tabular}{|c|c|c|}
\hline$A$ & $-A \pm 2 t A$ & $\# E_{A}^{0}\left(\mathbb{F}_{37}\right)$ \\
\hline $1,7,9,10,12,16,26,33,34$ & $q_{4}$ & 36 \\
\hline $3,4,11,21,25,27,28,30,36$ & $q_{2}$ & 40 \\
\hline $5,6,8,13,17,19,22,23,35$ & $q_{1}$ & 26 \\
\hline $2,14,15,18,20,24,29,31,32$ & $q_{1}$ & 50 \\
\hline
\end{tabular}

Corollary 2.14. Let $p \equiv 1(\bmod 12)$ be a prime and let $3 t^{2} \equiv 1$ $(\bmod p)$ with $t \in \mathbb{F}_{p}^{*}$. And let $g$ be a primitive root modulo $p$.

1. If $-1 \pm 2 t$ is a quartic residue in $\mathbb{F}_{p}$, then

$\# E_{1}^{0}: y^{2}=x^{3}+x \equiv 0(\bmod 3)$ and $\# E_{g^{2}}^{0}: y^{2}=x^{3}+g^{2} x \not \equiv 0$ $(\bmod 3)$.

2. If $-1 \pm 2 t$ is a quadratic residue but quartic non-residue in $\mathbb{F}_{p}$, then 
The number of points on elliptic curves $E_{A}^{0}: y^{2}=x^{3}+A x$ over $\mathbb{F}_{p} \bmod 24 \quad 99$

$\# E_{1}^{0}: y^{2}=x^{3}+x \not \equiv 0(\bmod 3)$ and $\# E_{g^{2}}^{0}: y^{2}=x^{3}+g^{2} x \equiv 0$ $(\bmod 3)$.

Proof. First, let $-1 \pm 2 t$ be a quartic residue in $\mathbb{F}_{p}$. Since $A=1$ is a quartic residue in $\mathbb{F}_{p}, 1 \cdot(-1 \pm 2 t)=-1 \pm 2 t$ is a quartic residue. By Lemma $2.12, \# E_{1}^{0}: y^{2}=x^{3}+x \equiv 0(\bmod 3)$. And $g^{2} \cdot(-1 \pm 2 t)$ is a quadratic residue but quartic non-residue in $\mathbb{F}_{p}$. So $\# E_{g^{2}}^{0}: y^{2}=x^{3}+g^{2} x \not \equiv$ $0(\bmod 3)$.

Secondly, let $-1 \pm 2 t$ be a quadratic residue but quartic non-residue in $\mathbb{F}_{p}$. Since $1 \cdot(-1 \pm 2 t)=-1 \pm 2 t$ is a quadratic residue but quartic non-residue in $\mathbb{F}_{p}, \# E_{1}^{0}: y^{2}=x^{3}+x \not \equiv 0(\bmod 3)$. And $g^{2} \cdot(-1 \pm 2 t)$ is a quartic residue in $\mathbb{F}_{p}$. So $\# E_{g^{2}}^{0}: y^{2}=x^{3}+g^{2} x \equiv 0(\bmod 3)$.

\section{Proof of Theorem 2.1}

By Proposition 2.2, Proposition 2.6, Corollary 2.9 and Lemma 2.12, the proof of Theorem 2.1 is complete.

Example 2.15. Let $p=7$, then $\# E_{A}^{0}\left(\mathbb{F}_{7}\right)=8$ for all $A \in \mathbb{F}_{7}$ is as the following table.

\begin{tabular}{|c|c|}
\hline $\mathrm{A}$ & $E_{A}^{0}\left(F_{7}\right)$ \\
\hline 1 & $O,(0,0),(1,3),(1,4),(3,3),(3,4),(5,2),(5,5)$ \\
\hline 2 & $O,(0,0),(4,3),(4,4),(5,3),(5,4),(6,2),(6,5)$ \\
\hline 3 & $O,(0,0),(1,2),(1,5),(2,0),(3,1),(3,6),(5,0)$ \\
\hline 4 & $O,(0,0),(2,3),(2,4),(3,2),(3,5),(6,3),(6,4)$ \\
\hline 5 & $O,(0,0),(2,2),(2,5),(3,0),(4,0),(6,1),(6,6)$ \\
\hline 6 & $O,(0,0),(1,0),(4,2),(4,5),(5,1),(5,6),(6,0)$ \\
\hline
\end{tabular}

\section{References}

[C] H. Cohen, A Course in Computational Algebraic Number Theory, Grad. Texts in Math., 138, Springer-verlag, Berlin, 1993, 198-199.

[D] L. E. Dickson, Criteria for the irreducibility of functions in a finite field, Bull. Amer. Math. Soc. 13(1906), 1-8.

[DISC] M. Demirci, Y. N. Ikikardes, G. Soydan and I. N. Cangul, Frey Elliptic Curves $E: y^{2}=x^{3}-n^{2} x$ on finite field $\mathbb{F}_{p}$ where $p \equiv 1(\bmod 4)$ is prime, to be printed.

[DSC] M. Demirci, G. Soydan and I. N. Cangul, Rational points on Elliptic Curves $E: y^{2}=x^{3}+a^{3}$ in $\mathbb{F}_{p}$ where $p \equiv 1(\bmod 4)$ is prime, Rocky Mountain Journal of Mathematics, 37, no 5, 2007.

[IR] K. Ireland and M. Rosen, A Classical Introduction to Modern Number Theory, Springer-Verlag, 1981.

[ISDBC] I. Inam, G. Soydan, M. Demirci, O. Bizim and I. N. Cangul, Corrigendum on "The Number of Points on Elliptic Curves $E: y^{2}=x^{3}+c x$ over $\mathbb{F}_{p} \bmod 8$ ", Commun. Korean Math. Soc. 22 (2007), no. 2, 207-208. 
[ISDC] N. Y. Ikikardes, G. Soydan, M. Demirci and I. N. Cangul, Classification of the Bachet Elliptic Curves $y^{2}=x^{3}+a^{3}$ in $\mathbb{F}_{p}$, where $p \equiv 1(\bmod 6)$ is Prime, Int. J. Math. Sci. (WASET) 1 (2007), no. 4, 239-241.

[ISDC2] N. Y. Ikikardes, G. Soydan, M. Demirci and I. N. Cangul, Rational points on Frey Elliptic Curves $E: y^{2}=x^{3}-n^{2} x$, Adv. Stud. Contemp. Math. (Kyungshang) 14 (2007), no. 1, 69-76.

[K] A. W. Knapp, Elliptic curves, Princeton Uinversity Press, New Jersey 1992.

[KKP] D. Kim, J. K. Koo and Y. K. Park, On the elliptic curve modulo p, Journal of Number Theory 128(2008), 945-953.

[PKL] H. Park, D. Kim and E. Lee, The numbers of points elliptic curves $E: y^{2}=$ $x^{3}+c x$ over $\mathbb{F}_{p} \bmod 8$, Commun. Korean Math. Soc. 18 (2003), 31-37.

[S] R. Schoof, Counting points on elliptic curves over finite fields, Journal de Theorie des Nomvres de Bordeaux, 7(1995), 219-254.

[Si] J. H. Silverman, The arithmetic of elliptic curves, Graduate Texts in Mathematics, 106. Springer-Verlag, New York, 1992.

[St] L. Stickelberger, Uber eine neue Eigenschaft der Diskriminanten algebraischer Zahlkorper, in:Verh. I. Internat. Math. Kongress, Zurich, 1987, 182-193.

H. S. Park

Department of Mathematics, Chonbuk National University, Chonju 561-756, Korea.

E-mail: park@jbnu.ac.kr

\section{S. H. You}

Department of Mathematics, Chonbuk National University, Chonju 561-756, Korea.

E-mail: m2zzang@hanmail.net

\section{Y. Kim}

Division of Fusion and Convergence of Mathematical Sciences, National Institute for Mathematical Sciences,

Dajeon 305-390, Korea.

E-mail: daeyeoul@nims.re.kr

M. H. Kim

Department of Mathematics, Chonbuk National University, 
The number of points on elliptic curves $E_{A}^{0}: y^{2}=x^{3}+A x$ over $\mathbb{F}_{p} \bmod 24101$

Chonju 561-756, Korea.

E-mail: minabout@hanmail.net 